\title{
Impact of squamous and/or glandular differentiation on recurrence and progression following transurethral resection for non-muscle invasive urothelial carcinoma of bladder
}

\author{
HAO XU ${ }^{1,2^{*}}$, LINGUO XIE ${ }^{1,2^{*}}$, XIAOTENG LIU ${ }^{1,2^{*}}$, YU ZHANG $^{1,2}$, ZHONGHUA SHEN $^{1,2}$, TAO CHEN $^{1,2}$,

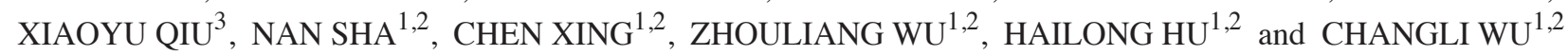 \\ ${ }^{1}$ Department of Urology; ${ }^{2}$ Tianjin Key Laboratory of Urology, Tianjin Institute of Urology, \\ Second Hospital of Tianjin Medical University, Hexi, Tianjin 300211; \\ ${ }^{3}$ College of Management and Economics, Tianjin University, Nankai, Tianjin 300072, P.R. China
}

Received September 2, 2015; Accepted March 3, 2017

DOI: $10.3892 / \mathrm{ol} .2017 .6581$

\begin{abstract}
The aim of the present study was to investigate the impact of squamous and/or glandular differentiation on the recurrence and progression in patients with nonmuscle invasive urothelial carcinoma of bladder (NMIUCB) following transurethral resection (TURBT). A total of 869 patients with NMIUCB who had been treated with TURBT at The Second Hospital of Tianjin Medical University (Tianjin, China) between January 2006 and January 2011 were retrospectively selected for the present analysis. Associations among squamous and/or glandular differentiation with other clinical and pathological features were assessed by the $\chi^{2}$ test. Recurrence-free survival (RFS) and progression-free survival (PFS) curves were estimated using the Kaplan-Meier method. Univariate and multivariate analyses were performed through a Cox's proportional hazards regression model. Among the 869 patients, 232 (26.7\%) patients had squamous and/or glandular differentiation. High grade tumors were more common in patients with squamous and/or glandular differentiation compared with those with pure urothelial carcinoma of bladder $(\mathrm{P}<0.001)$. Associations between age $(\mathrm{P}=0.115)$, sex $(\mathrm{P}=0.184)$, tumor size $(\mathrm{P}=0.223)$, tumor multiplicity $(\mathrm{P}=0.108)$, pathological tumor stage $(\mathrm{P}=0.909)$ and squamous and/or glandular differentiation were not observed to be statistically significant. There was
\end{abstract}

Correspondence to: Professor Hailong Hu or Professor Changli Wu, Department of Urology, Tianjin Institute of Urology, Second Hospital of Tianjin Medical University, 23 Pingjiang Road, Hexi, Tianjin 300211, P.R. China

E-mail: hhllove2004@163.com

E-mail: wujygc2003@163.com

${ }^{*}$ Contributed equally

Key words: squamous, glandular, urothelial carcinoma, recurrence, progression a significant tendency towards higher recurrence rate and shorter RFS time in patients with squamous and/or glandular differentiation. However, no statistically significant differences were observed in progression rate and PFS between the two groups. The multivariate Cox regression analysis, identified squamous and/or glandular differentiation as an independent prognostic predictor of recurrence (hazard ratio $=1.46,95 \%$ confidence interval $=1.10-1.92, \mathrm{P}=0.008)$. In the present study, the presence of squamous and/or glandular differentiation was associated with a higher recurrence rate and shorter RFS time in patients with NMIUCB. Squamous and/or glandular differentiation is therefore an independent prognostic predictor of recurrence.

\section{Introduction}

Bladder cancer is the 6th leading cause of new cancer cases and 9th leading cause of cancer-associated mortality in males worldwide. An estimated 429,800 new cases of bladder cancer and 165,100 bladder cancer associated mortalities occurred in 2012 worldwide (1). The histological types of bladder cancer are quite diverse, and the vast majority of cases are urothelial cancer (2). However, urothelial cancer is also known to have different variants, as determined by histological features. Squamous differentiation, defined by the presence of keratinization or intercellular bridges, is the most common variant of urothelial cancer, with previous studies noting its presence in 16.8-22.1\% of cases (3-5) (Fig. 1). Glandular differentiation, characterized by intratumoral tubular or enteric gland-like spaces, is less common compared with squamous differentiation, with an incidence of $\leq 16 \%$ (6) (Fig. 2). These two histological subtypes often coexist within the same tumor $(7,8)$.

It is controversial whether urothelial carcinoma of bladder (UCB) with squamous and/or glandular differentiation behave more aggressively compared with pure UCBs. Generally, squamous and/or glandular differentiation has been thought to have little clinical significance in urothelial carcinomas (9-11). However, previous studies have suggested that the presence of variant histology is usually associated with more aggressive behavior and a worse patient outcome $(3,12-14)$. The clinical 
management for patients with UCB with divergent histology remains controversial.

However, these aforementioned studies are largely limited to muscle invasive UCBs treated with radical cystectomy. The significance of squamous and/or glandular histological features, characterized at transurethral resection (TURBT), remains unclear $(15,16)$. The present study therefore aims to determine the prognostic relevance of squamous and/or glandular differentiation in patients with nonmuscle invasive urothelial carcinoma of bladder (NMIUCB) that have been treated with TURBT.

\section{Materials and methods}

Ethics statement. The present study was approved by the Ethics Committee of the Second Hospital of Tianjin Medical University (Tianjin, China). The retrospective study conducted did not affect the clinical course of any patient, and all patient records were analyzed anonymously. Due to the retrospective nature of the study, written informed consent for each individual was not necessary.

Patient characteristics. All clinical data were prospectively gathered from patient charts and electronic medical records. A total of 869 patients with NMIUCB who underwent TURBT between January 2006 and January 2011 were enrolled into the present study. The inclusion criteria for the study were: Patients who underwent TURBT as an initial treatment; a pathological diagnosis of tumors as NMIUCB [noninvasive papillary urothelial carcinoma (pTa) + tumor invading into the lamina propria (pT1)]; patients for whom a detailed assessment of primary tumor histology was available; and patients who received adjuvant intravesical chemotherapy following TURBT. Exclusion criteria for the present study include: An absence of urothelial carcinoma histology in the TURBT specimen; the presence of urethral or upper tract primaries, or distant metastasis at diagnosis; and the presence of carcinoma in situ (CIS) and other histological variants. For each patient, the following clinical factors were collected: Age, sex, tumor multiplicity, tumor size, pathological tumor stage and tumor grade. Tumor size was defined as being the greatest diameter observed during microscopic analysis of the surgical specimen.

Surgical procedure and pathological evaluation. All patients were treated with TURBT according to the following standardized procedure used by all surgeons in the present study. All visible tumors or suspicious mucosal lesions were resected with a monopolar loop electrode until the tumor base reached the deep muscle layer and transparently showed perivesical fat. Tissue samples were collected and fixed in formalin and embedded in paraffin. Available hematoxylin and eosin stained slides (staining for $5 \mathrm{~min}$ at $25^{\circ} \mathrm{C}$ with hematoxylin and $30 \mathrm{~s}$ at $25^{\circ} \mathrm{C}$ with eosin) of tumor tissue samples (thickness $4 \mu \mathrm{m}$ ) were reassessed using a light microscope (magnification, x100). The pathological stage and tumor grade were reassigned by a single genitourinary pathologist at the Second Hospital of Tianjin Medical University, according to the 2002 World Health Organization (WHO) tumor-node-metastasis classification of 6th American Joint Committee on Cancer and the 1973 WHO grading system, respectively $(17,18)$. Squamous

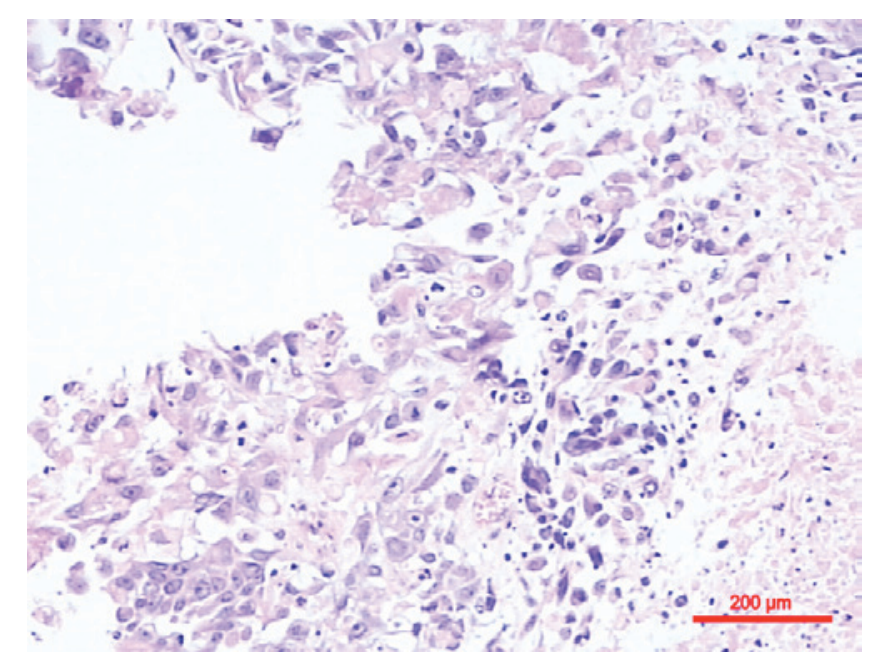

Figure 1. Urothelial carcinoma with squamous differentiation. Staining of the samples was performed using hematoxylin and eosin. Magnification, x100. Scale bar represents $200 \mu \mathrm{m}$.

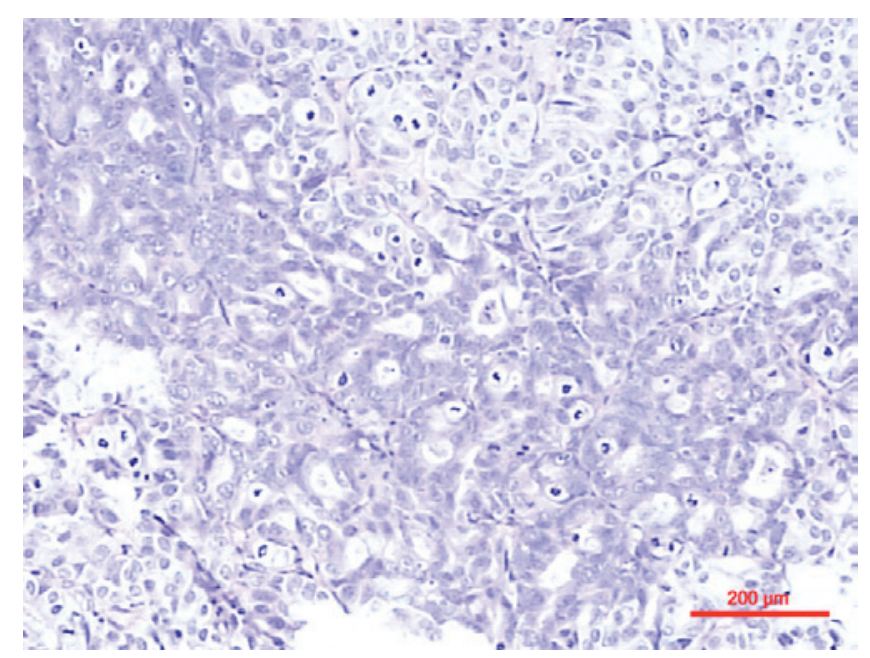

Figure 2. Urothelial carcinoma with glandular differentiation. Staining of the samples was performed using hematoxylin and eosin. Magnification, x100. Scale bar, $200 \mu \mathrm{m}$.

differentiation was defined by the presence of intercellular bridges or keratinization and glandular differentiation was defined as gland formation in the tumor with or without mucin production (19). According to the published classification recommendations, tumors with any urothelial carcinoma (UC) component as well as a second, nonurothelial malignant component were considered to represent UC with histological differentiation. Thus, patients with pure non UC were not included in the present study.

Initial adjuvant intravesical therapy. Due to the inaccessibility of the drug Bacillus Calmette-Guerin in China before 2015, all enrolled patients received the anthracycline antibiotic chemotherapy drugs including epirubicin or pirarubicin. The dosage and scheme used varied between patients as this observational study was based on a retrospective analysis. The schedule for instillation chemotherapy consisted of weekly instillations for 8 consecutive weeks and monthly instillations 
Table I. Clinicopathological patient demographics stratified by squamous and/or glandular differentiation in TURBT specimen.

\begin{tabular}{|c|c|c|c|}
\hline Clinicopathological features & Pure UCB no. (\%) & $\begin{array}{l}\text { UCB + squamous/glandular } \\
\text { differentiation }(\%)\end{array}$ & P-value \\
\hline No. of patients & 637 & 232 & \\
\hline Mean age at initial TURBT, years & $64.89 \pm 10.28$ & $66.16 \pm 11.02$ & \\
\hline$<65$ & $292(45.84)$ & $94(40.52)$ & 0.115 \\
\hline$\geq 65$ & $345(54.16)$ & $138(59.48)$ & \\
\hline \multicolumn{4}{|l|}{ Sex } \\
\hline Male & $525(82.42)$ & $182(78.45)$ & 0.184 \\
\hline Female & $112(17.58)$ & $50(21.55)$ & \\
\hline \multicolumn{4}{|l|}{ Tumor size, $\mathrm{cm}$} \\
\hline$<3$ & $437(68.60)$ & 149 (64.22) & 0.223 \\
\hline$\geq 3$ & $200(31.40)$ & $83(35.78)$ & \\
\hline \multicolumn{4}{|l|}{ Multiplicity } \\
\hline Single & $390(61.22)$ & $128(55.17)$ & 0.108 \\
\hline Multiple & 247 (38.78) & $104(44.83)$ & \\
\hline \multicolumn{4}{|l|}{ Pathological tumor stage } \\
\hline pTa & $37(5.81)$ & $13(5.60)$ & 0.909 \\
\hline pT1 & $600(94.19)$ & $219(94.40)$ & \\
\hline \multicolumn{4}{|l|}{ Tumor grade } \\
\hline Low & $452(70.96)$ & $96(41.38)$ & $<0.001$ \\
\hline High & $185(29.04)$ & $136(58.62)$ & \\
\hline
\end{tabular}

Data are presented as the mean \pm standard deviation. Tumor size was classified as being the largest diameter of the resected tumor. TURBT, transurethral resection; UCB, urothelial carcinoma of bladder; pTa, noninvasive papillary urothelial carcinoma; pT1, tumor invading into the lamina propria.

for 12 consecutive months. The chemotherapeutic drug used was either 40-60 mg epirubicin or $30 \mathrm{mg}$ pirarubicin.

Follow-up and clinical outcomes. Postoperative follow-up was conducted by rigid cystoscopy every 3 months for a period of 2 years, every 6 months thereafter until 5 years and then yearly according to the Chinese guidelines, which are the same as the US and European guidelines (20). The end points of the present study were recurrence and progression. Outcomes of interest in the present study were recurrence-free survival (RFS) and progression-free survival (PFS). The RFS period was estimated from the date of surgery to date of first clinical recurrence (any grade, any pathological stage of tumor or CIS). The PFS duration was calculated from the time of surgery to the date when the disease developed to a higher histological or pathological stage and/or to metastasis. For patients without recurrence and progression, the end point was the date of the last available follow-up cystoscopy.

Statistical analysis. Statistical analysis was performed using the statistical software SPSS, version 20 (IBM SPSS, Armonk, NY, USA). Continuous variables according to the presence of the squamous and/or glandular differentiation were compared using the independent sample t-test, and categorical variables were compared using the $\chi^{2}$ test. RFS and PFS curves were calculated by the Kaplan-Meier method and differences were analyzed by the log-rank test. Univariate and multivariate Cox proportional hazard analysis were performed to verify independent predictive parameters of recurrence and progression. All tests were 2 -sided, and $\mathrm{P}<0.05$ was considered to indicate a statistically significant difference.

\section{Results}

The clinicopathological demographics of patients with pure UCB, and those with UCB and squamous and/or glandular differentiation are presented in Table I. The mean age at initial TURBT was $64.89 \pm 10.28$ years in patients with pure UCB and $66.16 \pm 11.02$ years in patients with squamous and/or glandular differentiation. Among the 869 patients, 195 (22.4\%) had UCB with squamous differentiation, 27 (3.1\%) had glandular differentiation, and $10(1.2 \%)$ had squamous and glandular differentiation, which is similar to the results of a previous study (2). Age, sex, tumor size, tumor multiplicity and pathological tumor stage did not differ depending on the presence of the squamous and/or glandular differentiation. However, high grade tumors were more common in UCB with squamous and/or glandular differentiation compared with pure UCB (58.62 vs. 29.04\%, $\mathrm{P}<0.001$ ).

During the median 76.0 months follow-up, 89/232 patients with squamous and/or glandular differentiation and 149/637 patients with pure UCB experienced disease recurrence (Table II). Similarly, 27/232 patients with squamous and/or glandular differentiation and 72/637 patients with pure UCB 
Table II. Distribution of the two groups in accordance with recurrence and progression.

\begin{tabular}{lrrr}
\hline $\begin{array}{l}\text { Recurrence and } \\
\text { progression }\end{array}$ & $\begin{array}{c}\text { Pure UCB } \\
\text { no. (\%) }\end{array}$ & $\begin{array}{c}\text { UCB + squamous/ } \\
\text { glandular } \\
\text { differentiation }\end{array}$ & P-value \\
\hline $\begin{array}{l}\text { Recurrence } \\
\text { Yes }\end{array}$ & $149(23.39)$ & $89(38.36)$ & $<0.001$ \\
No & $488(76.61)$ & $143(61.64)$ & \\
$\begin{array}{l}\text { Mean RFS, } \\
\text { mo (range) }\end{array}$ & $93.01(2-114)$ & $80.54(3-113)$ & $<0.001$ \\
$\begin{array}{l}\text { Progression } \\
\text { Yes }\end{array}$ & $72(11.30)$ & $27(11.64)$ & 0.891 \\
No & $565(88.70)$ & $205(88.36)$ & \\
$\begin{array}{l}\text { Mean PFS, } \\
\text { mo (range) }\end{array}$ & $104.74(3-114)$ & $103.65(5-114)$ & 0.813 \\
\hline
\end{tabular}

UCB, urothelial carcinoma of bladder; RFS, recurrence-free survival; PFS, progression-free survival; mo, months.

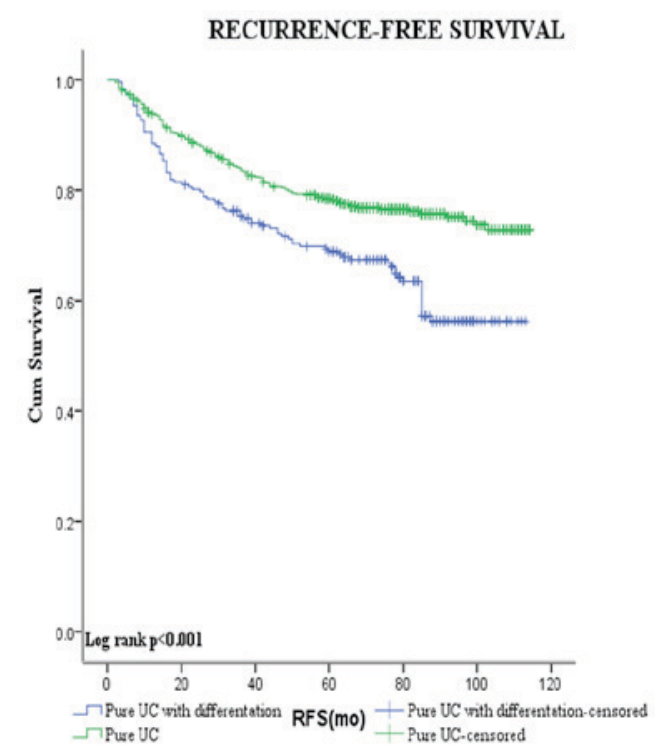

Figure 3. Kaplan-Meier curve of the recurrence-free survival rates for the two groups (log-rank test result: <0.001). Cum, cumulative; RFS, recurrence-free survival; mo, months; UC, urothelial carcinoma.

achieved disease progression. Patients with squamous and/or glandular differentiation were significantly more likely to recur compared with those with pure UCB (38.36 vs. $23.39 \%$, respectively, $\mathrm{P}<0.001)$. However, no statistically significant difference was observed in the rates of progression between patients with squamous and/or glandular differentiation and those with pure UCB (11.64 vs. $11.30 \%$, respectively, $\mathrm{P}=0.891$ ).

Figs. 3 and 4 show the Kaplan-Meier plots for recurrence-free and progression-free survival estimates stratified by pure UCB vs. those with squamous and/or glandular differentiation. Patients with squamous and/or glandular cell differentiated UCB had shorter mean RFS duration compared with those with pure UCB (80.5 vs. 93.0 months, respectively,

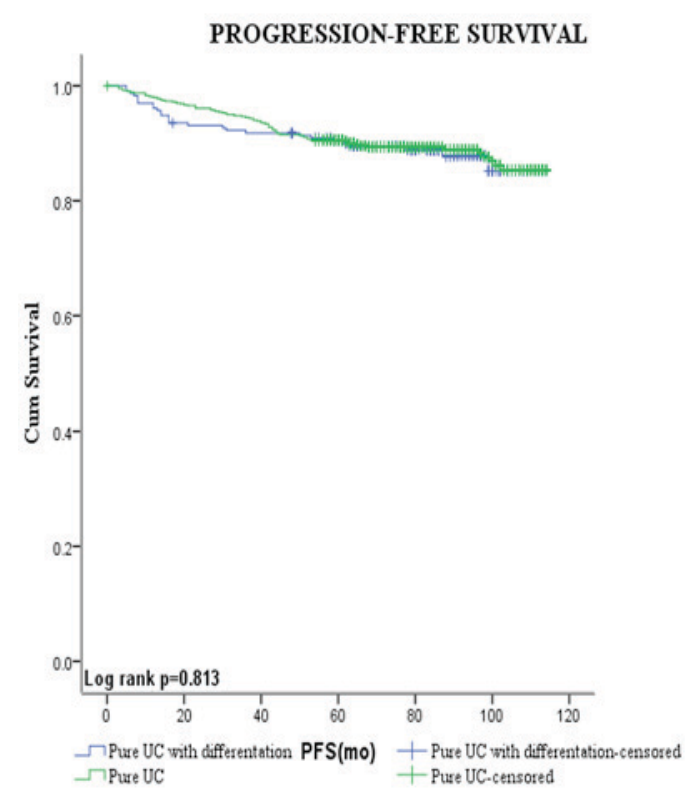

Figure 4. Kaplan-Meier curve of the progression-free survival rates for the two groups (log-rank test result: 0.813). Cum, cumulative; PFS, progression-free survival; mo, months; UC, urothelial carcinoma.

$\mathrm{P}<0.001)$. By contrast, no significant difference was observed between the PFS of the two groups (104.7 vs. 103.6 months, respectively, $\mathrm{P}=0.813$ ).

The present study conducted additional analysis using Cox's proportional hazard regression analysis to evaluate the role of each variable in recurrence (Table III). The results of univariate analysis revealed that tumor multiplicity [hazard ratio (HR) 1.86, 95\% confidence interval (CI) 1.44-2.40, $\mathrm{P}<0.001$ ], tumor size (HR 1.82, 95\% CI 1.41-2.35, $\mathrm{P}<0.001$ ), tumor grade (HR $1.78,95 \%$ CI $1.38-2.30, \mathrm{P}<0.001)$, pathological tumor stage (HR 2.44, 95\% CI 1.08-5.49, $\mathrm{P}=0.031$ ) and the presence of squamous and/or glandular differentiation (HR 1.72, 95\% CI 1.33-2.24, P<0.001) were significant factors associated with disease recurrence.

However, in multivariate Cox's proportional hazard regression analysis, only tumor multiplicity (HR 1.73, 95\% CI 1.34-2.24, $\mathrm{P}<0.001$ ), tumor size (HR 1.72, 95\% CI 1.33-2.22, $\mathrm{P}<0.001$ ), tumor grade (HR 1.42, 95\% CI 1.08-1.86, $\mathrm{P}=0.012$ ) and the presence of squamous and/or glandular differentiation (HR 1.46, 95\% CI 1.10-1.92, $\mathrm{P}=0.008$ ) were demonstrated to be significant independent predictors of recurrence.

\section{Discussion}

Bladder cancer comprises a wide range of histological types. UC is derived from uroepithelium, and is the most common type of bladder cancer accounting for $>90 \%$ of bladder cancer cases (2). UC is known to demonstrate variant histologic features, otherwise known as divergent differentiation. Squamous and glandular elements represent the most common types of divergent histology in primary UCB, and these features may coexist within a single tumor (2). However, the clinical significance of squamous and/or glandular differentiation remains uncertain $(11,12,14,21)$.

Generally, it has been considered that there are no prognostic differences between patients with UCB with and without 
Table III. Univariate and multivariate analyses according to recurrence.

\begin{tabular}{|c|c|c|c|c|}
\hline \multirow[b]{2}{*}{ Factor } & \multicolumn{2}{|c|}{ Univariate } & \multicolumn{2}{|c|}{ Multivariate } \\
\hline & HR $(95 \% \mathrm{CI})$ & P-value & HR $(95 \%$ CI $)$ & P-value \\
\hline \multicolumn{5}{|c|}{ Age, years } \\
\hline $\begin{array}{l}<65 \\
\geq 65\end{array}$ & $1.16(0.90-1.49)$ & 0.265 & - & - \\
\hline \multicolumn{5}{|l|}{ Sex } \\
\hline $\begin{array}{l}\text { Male } \\
\text { Female }\end{array}$ & $1.08(0.77-1.50)$ & 0.657 & - & - \\
\hline \multicolumn{5}{|c|}{ Multiplicity } \\
\hline $\begin{array}{l}\text { Single } \\
\text { Multipl }\end{array}$ & $1.86(1.44-2.40)$ & $<0.001$ & $1.73(1.34-2.24)$ & $<0.001$ \\
\hline \multicolumn{5}{|c|}{ Tumor size, $\mathrm{cm}$} \\
\hline $\begin{array}{l}<3 \\
\geq 3\end{array}$ & $1.82(1.41-2.35)$ & $<0.001$ & $1.72(1.33-2.22)$ & $<0.001$ \\
\hline \multicolumn{5}{|c|}{ Tumor grade } \\
\hline $\begin{array}{l}\text { Low } \\
\text { High }\end{array}$ & $1.78(1.38-2.30)$ & $<0.001$ & $1.42(1.08-1.86)$ & 0.012 \\
\hline $\begin{array}{l}\text { Patholog } \\
\text { pTa } \\
\text { pT1 }\end{array}$ & $2.44(1.08-5.49)$ & 0.031 & $2.04(0.90-4.60)$ & 0.088 \\
\hline \multicolumn{5}{|c|}{$\begin{array}{l}\text { Squamous and/or } \\
\text { glandular differentiation }\end{array}$} \\
\hline $\begin{array}{l}\text { Absent } \\
\text { Present }\end{array}$ & $1.72(1.33-2.24)$ & $<0.001$ & $1.46(1.10-1.92)$ & 0.008 \\
\hline
\end{tabular}

HR, hazard ratio; CI, confidence interval; pTa, noninvasive papillary urothelial carcinoma; pT1, tumor invading into the lamina propria.

differentiation (9-11). Kim et al (11) conducted a retrospective review of 1,013 patients who had undergone radical cystectomy, and reported that patients with urothelial carcinoma, and squamous and/or glandular differentiation were more likely to possess extravesical tumors and a node positive disease. Multivariate analysis controlling for clinicopathological variables revealed that squamous and/or glandular differentiation was not significantly associated with the risk of mortality from bladder cancer (11). Similarly, in a single referral center study of 2,444 patients who had undergone radical cystectomy with extended lymph node dissection, the outcomes of patients with UCB with squamous and/or glandular differentiation were similar to those of patients with pure UCB, considering comparable demographic, clinicopathological and management characteristics (22).

However, other studies contradict these findings, indicating that squamous and/or glandular differentiation appears to be an unfavorable prognostic feature in such patients undergoing radical cystectomy, which is possibly due to its association with high grade tumors $(3,4,13,21,23,24)$. Squamous and/or glandular differentiation is usually found in moderate to high grade tumors, and often has deeply invasive behavior. Antunes et al (3) identified that squamous differentiation was an independent prognostic factor for cancer specific survival in patients with bladder cancer that had been treated with radical cystectomy. In addition, Honma et al (12) reported that the existence of a squamous cell carcinoma component in the specimen may result in a strong impact on the development of local recurrence following radical cystectomy.

Treatment options for patients with UCB with squamous and/or glandular differentiation is currently debated. Previous studies have suggested that these variants may be more resistant to chemotherapy and radiation therapy compared with pure UCB $(13,19,25)$. However, Scosyrev et al $(10)$ reported that patients with squamous or glandular histological UCB variants exhibited an improved response to neoadjuvant chemotherapy [methotrexate, vinblastine, doxorubicin (Adriamycin) and cisplatin] in a post hoc analysis of a prospective trial. Additional studies are required in order to evaluate the role of chemotherapy and radiation therapy in UCBs with squamous and/or glandular differentiation.

The effect of squamous and/or glandular differentiation in transurethral resections of the bladder on prognosis also remains unclear. Billis et al (15) reported that urothelial bladder carcinomas with squamous and/or glandular differentiation were more aggressive neoplasms compared with pure UCBs. A statistically significant association was observed between higher stage and differentiation in two groups of 
urothelial carcinoma with and without squamous and/or glandular differentiation (15). Erdemir et al (23) reported that squamous and/or glandular differentiation was significantly associated with higher pathological stage and histological grade. Patients with variant UCB histology had a higher risk of disease recurrence and progression, and lower survival rates following TURBT compared with patients with pure UCB.

A limited number of studies have investigated the impact of squamous and/or glandular differentiation on oncologic outcomes in non-invasive UCB. In a study by Miller and Epstein (25), the presence of glandular differentiation in noninvasive UCB was associated with an increased risk of developing to high-grade prognostically poor invasive bladder carcinomas including poorly-differentiated UCB.

Unlike previous studies, the emphasis of the present study was to investigate the impact of squamous and/or glandular differentiation on recurrence and progression rates in patients with NMIUCB following TURBT. All patients enrolled in the present study were pathologically diagnosed as having NMIUCB (pTa + pT1) and TURBT was performed as the initial treatment. The results demonstrated that patients with squamous and/or glandular differentiation had a higher recurrence rate and shorter mean RFS duration compared with patients with pure UC. However, no statistically significant difference was observed between the incidence of progression and mean PFS duration.

Multivariate Cox regression analysis revealed that squamous and/or glandular differentiation, tumor size, tumor count and tumor grade were independent prognostic factors of RFS. The most notable finding was the grade distribution of the tumors with differentiation. Patients with differentiation were significantly more likely to possess high grade tumors compared with those with pure UCB. These findings suggested that the presence of squamous and/or glandular differentiation in patients with NMUCB is associated with more aggressive behavior. Follow-up should therefore be closer for patients with squamous and/or glandular differentiation due to a higher risk of recurrence.

The present study was not devoid of limitations. Firstly, limitations occurred due to the retrospective nature of the present study, with there being nonrandomized samples from a single institution. In addition, a re-review of all specimens was not performed and therefore the present study relied on the pathologist to identify and report the variant histologies. Furthermore, the present study did not investigate the impact of squamous and glandular differentiation on NMUCB individually which may have a disparate prognostic importance.

In summary, the presence of squamous and/or glandular differentiation in NMIUCB identified at TURBT indicates a locally aggressive and advance disease. As an independent prognostic factor of recurrence, patients with the variant form of NMIUCB present a shorter RFS duration, and should therefore be followed up closely in case of recurrence.

\section{Acknowledgements}

The present study was funded by grants from The Natural Science Foundation of Tianjin (grant no. 14JCYBJC26300) and National Key Specialty Construction of Clinical Projects, The Natural Science Foundation of Tianjin (grant no. 15JCYBJC24600), and Tianjin Major Scientific and Technological special Project (grant no. 12ZCDZSY16900). The authors would like to thank all the study participants, urologists and study coordinators for their participation in the present study.

\section{References}

1. Torre LA, Bray F, Siegel RL, Ferlay J, Lortet-Tieulent J and Jemal A: Global cancer statistics, 2012. CA Cancer J Clin 65: 87-108, 2015.

2. Chalasani V, Chin JL and Izawa JI: Histologic variants of urothelial bladder cancer and nonurothelial histology in bladder cancer. Can Urol Assoc J 3 (6 Suppl 4): S193-S198, 2009.

3. Antunes AA, Nesrallah LJ, Dall'Oglio MF, Maluf CE, Camara C, Leite KR and Srougi M: The role of squamous differentiation in patients with transitional cell carcinoma of the bladder treated with radical cystectomy. Int Braz J Urol 33: 339-346, 2007.

4. Yang MH, Yen CC, Chen PM, Wang WS, Chang YH, Huang WJ, Fan FS, Chiou TJ, Liu JH and Chen KK: Prognostic-factors-based risk-stratification model for invasive urothelial carcinoma of the urinary bladder in Taiwan. Urology 59: 232-239, 2002.

5. Lopez-Beltran A, Requena MJ, Alvarez-Kindelan J, Quintero A, Blanca A and Montironi R: Squamous differentiation in primary urothelial carcinoma of the urinary tract as seen by MAC387 immunohistochemistry. J Clin Pathol 60: 332-335, 2007.

6. Domanowska E, Jozwicki W, Domaniewski J, Golda R, Skok Z, Wiśniewska H, Sujkowska R, Wolski Z and Jozwicka G: Muscle-invasive urothelial cell carcinoma of the human bladder: Multidirectional differentiation and ability to metastasize. Hum Pathol 38: 741-746, 2007.

7. Grace DA and Winter CC: Mixed differentiation of primary carcinoma of the urinary bladder. Cancer 21: 1239-1243, 1968.

8. Fegen JP, Albert DJ and Persky L: Adenocarcinoma and transitional cell carcinoma occurring simultaneously in the urinary bladder (mixed tumor). J Surg Oncol 3: 387-392, 1971.

9. Kastritis E, Dimopoulos MA, Antoniou N, Deliveliotis C, Chrisofos M, Skolarikos A, Gika D and Bamias A: The outcome of patients with advanced pure squamous or mixed squamous and transitional urothelial carcinomas following platinum-based chemotherapy. Anticancer Res 26: 3865-3869, 2006.

10. Scosyrev E, Ely BW, Messing EM, Speights VO, Grossman HB, Wood DP, de Vere White RW, Vogelzang NJ, Trump DL, Natale RB, et al: Do mixed histological features affect survival benefit from neoadjuvant platinum-based combination chemotherapy in patients with locally advanced bladder cancer? A secondary analysis of southwest oncology group-directed intergroup study (S8710). BJU Int 108: 693-699, 2011.

11. Kim SP, Frank I, Cheville JC, Thompson RH, Weight CJ, Thapa $P$ and Boorjian SA: The impact of squamous and glandular differentiation on survival after radical cystectomy for urothelial carcinoma. J Urol 188: 405-409, 2012.

12. Honma I, Masumori N, Sato E, Takayanagi A, Takahashi A, Itoh N, Tamagawa M, Sato MA and Tsukamoto T: Local recurrence after radical cystectomy for invasive bladder cancer: An analysis of predictive factors. Urology 64: 744-748, 2004.

13. Frazier HA, Robertson JE, Dodge RK and Paulson DF: The value of pathologic factors in predicting cancer-specific survival among patients treated with radical cystectomy for transitional cell carcinoma of the bladder and prostate. Cancer 71: 3993-4001, 1993.

14. Jozwicki W, Domaniewski J, Skok Z, Wolski Z, Domanowska E and Jozwicka G: Usefulness of histologic homogeneity estimation of muscle-invasive urinary bladder cancer in an individual prognosis: A mapping study. Urology 66: 1122-1126, 2005.

15. Billis A, Schenka AA, Ramos CC, Carneiro LT and Araujo V: Squamous and/or glandular differentiation in urothelial carcinoma: Prevalence and significance in transurethral resections of the bladder. Int Urol Nephrol 33: 631-633, 2001.

16. Mazzucchelli R, Morichetti D, Lopez-Beltran A, Cheng L, Scarpelli M, Kirkali Z and Montironi R: Neuroendocrine tumours of the urinary system and male genital organs: Clinical significance. BJU Int 103: 1464-1470, 2009.

17. Sobin LH and Wittekind C: TNM Classification of Malignant Tumours. Wiley-Liss, 2002.

18. Mostofi FK, Sobin LH and Torlini H: Histologic typing of urinary bladder tumours. World Health Organization, Geneva, pp1-20, 1973. 
19. Coulson WF: Clinical importance of squamous metaplasia in invasive transitional cell carcinoma of the bladder. J Clin Pathol 42: 1227-1228, 1989.

20. Babjuk M, Burger M, Zigeuner R, Shariat SF, van Rhijn BW, Compérat E, Sylvester RJ, Kaasinen E, Böhle A, Palou Redorta J, et al: EAU guidelines on non-muscle-invasive urothelial carcinoma of the bladder: Update 2013. Eur Urol 64: 639-653, 2013

21. Mazzucchelli L, Bacchi M, Studer UE, Markwalder R, Sonntag RW and Kraft R: Invasion depth is the most important prognostic factor for transitional-cell carcinoma in a prospective trial of radical cystectomy and adjuvant chemotherapy. Int J Cancer 57: 15-20, 1994

22. Shapur NK, Katz R, Pode D, Shapiro A, Yutkin V, Pizov G, Appelbaum L, Zorn KC and Duvdevani M: Is radical cystectomy mandatory in every patient with variant histology of bladder cancer. Rare Tumors 3: e22, 2011.
23. Erdemir F, Tunc M, Ozcan F, Parlaktas BS, Uluocak N, Kilicaslan I and Gokce O: The effect of squamous and/or glandular differentiation on recurrence, progression and survival in urothelial carcinoma of bladder. Int Urol Nephrol 39: 803-807, 2007.

24. Logothetis CJ, Dexeus FH, Chong C, Sella A, Ayala AG, Ro JY and Pilat S: Cisplatin, cyclophosphamide and doxorubicin chemotherapy for unresectable urothelial tumors: The M.D. Anderson experience. J Urol 141: 33-37, 1989.

25. Miller JS and Epstein JI: Noninvasive urothelial carcinoma of the bladder with glandular differentiation: Report of 24 cases. Am J Surg Pathol 33: 1241-1248, 2009. 